\title{
Gestión educativa para la participación activa de la familia en el rendimiento académico en el Liceo de Colorado de Abangares
}

\author{
Esteban Sánchez-Salazar \\ Estudiante de Licenciatura en Administración Educativa, \\ Universidad Estatal a Distancia, San José, Costa Rica \\ esanchez88@gmail.com
}

Aceptado: 03 de abril del 2019

\begin{abstract}
Resumen
El artículo analiza las acciones de la gestión educativa que refuerzan el apoyo de la familia al estudiantado de noveno año para mejorar su rendimiento académico. El estudio se realiza por el bajo rendimiento observado en las pruebas de bachillerato en el Liceo de Colorado. Para comprender la problemática se debe tener en cuenta una serie de factores externos e internos que afectan dicho rendimiento académico como, por ejemplo, la situación socioeconómica, el apoyo de la familia, la gestión realizada por el centro educativo, entre otras. Entre los principales resultados se encontró que la gestión educativa se encuentra en una etapa en la que no se ha integrado a la familia y realiza una colaboración pasiva y asistencialista, la cual no beneficia el rendimiento académico. Por eso, el gestor educativo tiene la labor de planear e implementar estrategias que impulsen la integración familiar al centro educativo con una participación activa con el fin de incrementar el rendimiento del alumnado.
\end{abstract}

Palabras clave: Gestión educativa; rendimiento académico; participación de los padres; familia; participación activa.

\section{Abstract \\ Educational management for the active participation of the family in academic performance at the Liceo de Colorado de Abangares}

The article analyzes the educational management actions that reinforce the support of the family to ninth-year students to improve their academic performance. The study is carried out due to the low performance observed in high school exams at the Liceo de Colorado. To understand the problem, a series of external and internal factors that affect the academic performance must be taken into account, such as, for example, the socioeconomic situation, the support of the family, the management carried out by the educational center, among others. Among the main results, it was found that educational management is at a stage in which the family has not been integrated and makes a passive and assistance-based collaboration, which does not benefit academic performance. Therefore, the educational manager has the task of planning and implementing strategies that promote family integration to the educational center with active participation in order to increase student performance

Key words: Educational management; academic achievement parent participation; family; active participation. 


\section{INTRODUCCIÓN}

La institución educativa y el personal docente administrativo son un ente abierto a la sociedad, lo cual significa que una institución trabaja por y para la comunidad en la que se encuentra. En este sentido, la educación es un asunto que concierne y es responsabilidad de todas las personas que habitan en una zona determinada. El Liceo de Colorado se encuentra inmerso en el contexto sociocultural de zona rural, donde hay pocas actividades laborales, las cuales se limitan a la pesca, el camaroneo y la empresa productora de cemento y derivados. Otras opciones son fuera de Colorado, en lugares cercanos como Limonal o las Juntas de Abangares. Estas circunstancias dificultan la cumilnación del proceso educativo.

En la actualidad, según el Sexto informe estado de la educación (2017), Costa Rica presenta un problema en la promoción de personas que logran concluir la secundaria. El informe antes citado indica que, durante los años 2010 y 2016, se incrementó de 45\% a 50,4\% la cantidad de personas que concluyeron el bachillerato, en edades entre los 18 a 22 años; pero aun así el porcentaje es muy bajo comparado con otros países de América Latina donde se alcanza $80 \%$. Señala también que la dificultad para incrementar la promoción del estudiantado que termina la secundaria se encuentra en el bajo índice de personas que logran concluir el noveno año.

Dentro de las circunstancias que impiden lograr el objetivo anterior se encuentra el bajo rendimiento, además, "el clima educativo y el ingreso de los hogares de donde provienen los estudiantes" (Sexto informe estado de la educación, 2017, p. 51) son algunos factores que influyen en el alumnado. Por lo anterior, es importante analizar cuáles acciones realiza la gestión educativa para reforzar el apoyo de la familia al estudiantado.

La gestión educativa es la encargada de mediar todos los procesos del centro educativo que integra diversos ámbitos, como, por ejemplo, personal docente, familia, alumnado, entre otros. Rico (2016) afirma que "es entendida como un proceso organizativo y orientado a la optimización de procesos y proyectos internos de las instituciones con el objetivo de perfeccionar los procedimientos pedagógicos, directivos, comunitarios y administrativos que en ella se movilizan" (p. 57).

Desde esta perspectiva, es necesario abordar la dimensión comunitaria, ya que para la persona gestora educativa es fundamental concretar acciones que refuercen el apoyo de la familia en el liceo. Tal y como señala Rico (2016) "la gestión educativa está orientada a permitir la integración de todos los actores de la institución, desde una perspectiva participativa y encaminada hacia la toma de decisiones óptimas y direccionadas hacia propósitos que aporten al mejoramiento de la educación" (p. 60). Es así que la persona administradora debe conocer el contexto cultural, social y demográfico del pueblo en el que se encuentra, ya que solamente así puede entender la realidad y las problemáticas presentes en la institución.

Asimismo, la persona directora debe comprender los diversos factores que afectan el rendimiento académico, el cual muchas veces es considerado solamente como la suma de notas obtenidas de los diferentes criterios de evaluación, esto significa que se centra en un sistema sumativo y no formativo. Los factores que influyen se pueden clasificar como externos e internos. Los primeros se refieren a situaciones socioeconómicas, familiares y culturales; los segundos están relancionados con las diferentes formas de aprendizaje y las capacidades del alumnado.

Por tanto, la familia es un elemento muy importante en el proceso de enseña-aprendizaje, ya que es la encargada, en primera instancia, de dar apoyo, tal y como lo dice el Código de la Niñez y la Adolescencia: "La obligación de procurar el desarrollo integral de la persona menor de edad les corresponde, en forma primordial, a los padres o encargados" (Diario Oficial la Gaceta, 1998, artículo 7). Sin embargo, hay diferentes formas en las que las familias cumplen su obligación. En lo que respecta a la participación familiar en el ámbito educativo puede catalogarse en: pasiva y activa. La primera, se enfoca en un asistencialismo por parte de las personas encargadas, o sea, una colaboración que se basa en aportes económicos, 
materiales o la simple asistencia a reuniones. Por el contrario, la participación activa, nos dice la UNESCO (2004) es "la posibilidad de incidir, opinar, aportar y discernir" (p. 26), en el proceso educativo, esto significa que la familia se empodera de la educación de los menores de edad.

Para lograr un empoderamiento se debe realizar un trabajo integral, en este sentido, Rico (2016) afirma que "la gestión educativa desde esta perspectiva no solo observa desde la parte interna, sino que debe estar conectada a factores externos que complementan estos procesos dentro de la institución" (p. 58). Se desprende que una de las labores del administrador educativo es servir de enlace entre la institución y la familia con el fin de incentivar la participación activa y que el proceso de enseñanza-aprendizaje sea un trabajo integral.

De ahí la importancia de analizar la gestión educativa que refuerza el empoderamiento de las familias hacia una participación activa dentro del centro educativo, con el fin de mejorar el rendimiento académico del alumnado. En esta investigación se trabaja con la población de noveno año, ya que como se señaló es una etapa importante en la conclusión del proceso de enseñanza-aprendizaje.

\section{METODOLOGÍA}

La investigación se fundamentó en un paradigma de investigación naturalista fenomenológico de un enfoque cualitativo, puesto que dibujó y analizó las diversas relaciones de los sujetos en su contexto. De este modo, se profundizó y describió la realidad de todos los sujetos y como estos interactúan y cambian su realidad. En este sentido, la investigación tiene como fin una descripción desde la situación en la que se desenvuelven los sujetos.

Para la recolección de datos se aplicaron entrevistas a profundidad, las cuales fueron aplicadas al director del centro educativo, a la orientadora y a la profesora guía. La intención de la entrevista a profundidad fue generar un diálogo abierto entre entrevistador y entrevistado con el propósito de crear confianza y conocer de forma más cercana la realidad de los sujetos. También, se realizó un cuestionario a las personas familiares y a los estudiantes de la sección 9-1 para conocer la opinión o problemas de los sujetos en el contexto del Liceo de Colorado.

Se trabajó con tres categorías: la gestión administrativa que refuerza el apoyo de la familia, el apoyo brindado al estudiantado desde la familia y la participación familiar. Cuando se finalizó con la obtención de información de los sujetos, se procedió a un análisis descriptivo de la realidad y del contexto de los sujetos de la investigación.

De este modo, un acercamiento fenomenológico junto con las categorías de análisis utilizadas pretendió conseguir resultados integrales con el fin de reflexionar sobre el entretejido en el que se desenvuelven todos los sujetos de la investigación. Por tanto, los resultados obtenidos se dividieron por áreas (director, docentes, estudiantado y familia) para finalmente contrastarlas y relacionarlas con la teoría.

\section{RESULTADOS}

La gestión es la responsable de construir puentes entre el centro educativo y la familia. Esta unidad es de suma importancia para poder adaptarse a los procesos de cambio y mejora que implica el accionar de un ente académico, donde deben trabajar todas las personas que están involucradas directamente en el proceso de enseñanza y aprendizaje. Por eso, es el director el principal encargado de dar voz y considerar los diferentes criterios de todos los miembros relacionados. Rico (2016) indica: "La gestión educativa se consolida, bajo dispositivos de cambio, integración, participación, dirección, organización 
y una evaluación de retroalimentación permanente" (p. 60). De ahí, el valor de la integración y el empoderamiento de las personas encargadas, ya que enriquece la discusión por la calidad de la educación.

Se debe recalcar que el gestor educativo es el encargado de ejecutar las diversas acciones para integrar todos los componentes, tanto internos como externos. La UNESCO (2011) señala que el gestor tiene que lidiar con "todos los elementos internos y externos, coexisten, interactúan y se articulan entre sí, de manera dinámica, en ellos se puede distinguir diferentes acciones, que puede agruparse según su naturaleza" (p. 32). Por eso, dentro de la dimensión institucional de la gestión educativa, se deben organizar todos los elementos para realizar estrategias que acerquen a las familias y trabajar de forma integral para mejorar el rendimiento académico.

Las reuniones con las familias son un instrumento muy importante para el director, pues es un medio idóneo para conocer la realidad y las problemáticas propias del estudiantado del centro educativo. Cuando el administrador educativo del Liceo de Colorado convoca reuniones estas le permiten obtener información importante para planificar sus estrategias. En el contexto del Liceo de Colorado, el director idea formas para acercar a las familias a la institución, ya que presentan muchas limitaciones debido a la ubicación geográfica y la situación socioeconómica. Por eso, una de las acciones para dar mayor oportunidad de participar en las reuniones es adaptarlas a horas de la tarde y ofrecer servicio de transporte.

Junto con las acciones antes mencionadas, el director, en coordinación con la persona docente guía, realiza un pequeño convivio donde se ofrece café o fresco con algún bocadillo, con el fin de incentivar el acercamiento de las familias al centro educativo. En palabras del gestor educativo: "Por medio de este tipo de actividades se puede motivar a las familias, ellas están acostumbradas a ir al liceo a recibir notas o escuchar sobre el comportamiento de su hija o hijo, por lo que, muchas veces no les resulta tan atractivo" (L. A. Gamboa, comunicación personal, 23 de mayo, 2018).

Así bien, dentro de las acciones realizadas por el director para mejorar el rendimiento académico, se puede insistir en un conocimiento del contexto en el que se desenvuelven las personas adolescentes y las circunstancias que complican la integración de las familias al liceo. También, dentro de las estrategias tomadas resalta la importancia de la comunicación como método para fortalecer las relaciones de todos los actores. No obstante, faltan acciones más directas por parte de la administración para integrar a las familias con la institución para lograr un trabajo colaborativo y una participación activa que ayude a mejorar el rendimiento académico.

Según la Declaración de Cochabamba (UNESCO, 2001), es responsabilidad del Estado integrar de forma activa a toda la comunidad al centro educativo, "es condición necesaria para aumentar la participación de la comunidad en la educación que el Estado asuma un efectivo liderazgo estipulando la participación de la sociedad en diseño, la ejecución y evaluación de impacto de las políticas educativas" (p. 7). En este caso, el Liceo de Colorado, como representante del Estado, es el encargado de articular la relación entre los miembros externos y el centro educativo.

Por otra parte, la familia es sumamente importante para la buena obtención de resultados en los estudios, ya que es en el hogar donde se puede encontrar apoyo, cariño, motivación, valores y otros recursos imprescindibles para el alumnado a la hora de afrontar dificultades. En este sentido, Oliva \& Villa (2013) afirman que la familia influye en aspectos tales como la biología, la psicología, la economía y la sociología.

Tras los cambios que ha sufrido la organización familiar con el pasar de los años, que las mujeres tengan cada vez más un papel protagonista en el hogar no es de extrañar, pues, según lo indica Barquero (2004) en su informe final sobre el Estado de la Nación, desde el año 1987 hasta el 2002 se ha dado un aumento en la cantidad de mujeres como jefas de hogar. Lo anterior es vinculante porque la mayoría de las personas que respondieron las encuestas de la investigación fueron mujeres, aunque casi la totalidad del alumnado vivía con madre y padre. Asimismo, el rol de la madre en la vida escolar es protagónico, 
pues en todas las preguntas el alumnado respondió que es la mamá quien les ayuda a estudiar, a realizar tareas o es quien está pendiente de lo que acontence en el centro educativo.

Sin embargo, cuando se les preguntó sobre las acciones concretas que realiza la persona encargada en asuntos escolares en el hogar, destacaron la poca ayuda en tareas y trabajos extra clase aunque estén pendientes de estos. Una tendencia similar se mantuvo cuando se encuestó sobre el apoyo brindado para estudiar o repasar la materia antes del examen. Lo anterior se reafirmó cuando se les interrogó sobre el apoyo en la casa y la mayoría exteriorizó que estudiaban solas, solamente una indicó que la madre le ayudaba y a otra persona el hermano. Al comparar los resultados de los datos recabados por ambos grupos, resaltó la falta de apoyo, lo cual motiva a pensar que si bien no hay abandono, tampoco hay acompañamiento pleno.

Suele darse compañía en la vida académica durante los primeros años de vida, pero conforme pasa el tiempo y se llega a grados superiores, como en secundaria, se confunde la independencia que ocupa una adolescente, con el alejamiento en sus asuntos escolares. Y, tal y como señala la Declaración de Cochabamba (UNESCO, 2001), a la que Costa Rica está subscrita, "la formación de los padres y madres, como primeros educadores de sus hijos, ha de constituir una estrategia fundamental en los programas de la primera infancia" (p. 19). Se puede agregar que, si se les pudiera educar a las personas encargadas sobre la relevancia de la participación en el proceso de enseñanza-aprendizaje, la integración al centro educativo sería más exitosa.

En este sentido, la presencia de las personas encargadas en el centro educativo es muy importante, pero no es sinónimo de mejor rendimiento. Es necesario que las reuniones sean más que un asunto informativo donde se entregan notas y reportes de conducta. Por eso, los llamados deben servir para exponer un diagnóstico de las herramientas con que cuentan las madres y padres de familia para ayudar a sus hijas o hijos, y mediante la información realizar capacitaciones para lograr mejorar el rendimiento académico por medio de un trabajo en conjunto de todo el centro educativo.

Existen diversas actividades que pueden realizar las familias para involucrarse en la educación de sus hijas e hijos. La UNESCO (2004) clasifica la participación de la familia en diversos tipos, como, por ejemplo, económica, asistencial o activa. Ahora bien, todos los tipos de participación son importantes y necesarios para un buen funcionamiento del centro educativo, pero, cuando se habla de participación activa, la familia se vuelve responsable directa de la educación de su hija o hijo mediante un empoderamiento que abarca la colaboración económica y de asistencia.

Así, es importante enfocarse en el tipo de acciones que realizan las familias del Liceo de Colorado, pues se pueden catalogar como participación pasiva, ya que se limitan a asistir a reuniones, participar en bingos o rifas, entre otras. Aun así, cuando se les pregunta a la familia y al alumnado sobre qué tipo de actividades podría ayudar a mejorar el rendimiento académico ambos grupos señalan que sería valioso que se realicen convivios y actividades recreativas para que puedan tener un espacio donde compartir.

En esta misma línea, se obtuvo una respuesta positiva por parte de las personas encuestadas sobre la importancia de la comunicación, pero el gestor educativo debe reforzar esas acciones para pasar de la comunicación monológica a la comunicación dialógica para la acción. Esto significa que, se debe dejar atrás el papel de informador (notas o conducta) y avanzar al rol de integrador para una participación activa.

En términos generales, las familias encuestadas de la sección 9-1 cumplen una participación pasiva en su integración con el centro educativo, lo cual se corrobora con las encuestas a profundidad en donde predomina el asistencialismo como forma de involucramiento. También, uno de los factores por tomar en cuenta para iniciar la integración de la familia es el papel de la mujer como cabeza de hogar y responsable de la casa, ya que complica más su acercamiento y participación activa en la institución, tal y como menciona Ramírez \& Rosés (2005) las mujeres jefas de hogar "enfrentan mayores dificultades, entre ellas, 
la incorporación y acceso al mercado laboral, así como en muchos casos no contar con el aporte económico del cónyuge para ellas y sus hijos e hijas" (p.47). La problemática mencionada multiplica las tareas tanto dentro del hogar como fuera de él, lo que provoca el recargo de los deberes en una sola persona.

En otros resultados obtenidos por medio una entrevista a profundidad aplicada a la orientadora y a la profesora guía de la sección 9-1, se comenta que la asistencia a reuniones de las personas encargadas no es la mejor, pues "siempre llegan las mismas personas". Cuando se indaga sobre la razón de la baja asistencia, las respuestas obtenidas son que la familia "por asuntos laborales o por falta de tiempo" no se puede comprometer en cuestiones académicas. Tal y como se señaló, cuando las labores y la responsabilidad de la familia corresponde a solo una persona, en este caso a la mujer, la capacidad para integrarse al proceso de enseñanza se obstaculiza.

Lo anterior no significa que las personas encargadas abandonen a su hija o hijo, según señala la orientadora, el apoyo que brindan no se relaciona directamente con horarios de estudios, más bien, están presentes en la parte económico o emocional, la cual es significativa para crear un buen clima de estudio en la casa, pero es necesario empoderar a las madres y los padres para conseguir una participación activa.

Ahora bien, cuando se interrogó a la orientadora y a la docente guía sobre qué acciones pueden motivar a las familias a apoyar a sus hijas o hijos, estas respondieron que "la administración debe tener un mayor acercamiento a los hogares para poder dar un mejor seguimiento de los problemas, dado que, aunque sí existe una buena comunicación con las personas encargadas muchas veces es solo cuando surge un problema" (C. E. Díaz y M. E. Marín, comunicación personal, 23 de mayo, 2018). En este sentido, es importante insistir en la necesidad de la comunicación constante y fluida que debe existir en un centro educativo. La UNESCO (2011) afirma la importancia del diálogo en la gestión administrativa y define comunicación como: "Capacidad de generar y mantener conversaciones para la acción" (p. 21), es decir, se tiene que dialogar no para comunicar, sino para actuar.

Para finalizar, entre los principales hallazgos se señala la necesidad de realizar acciones concretas por parte de la administración para trabajar el rendimiento académico desde un enfoque integral; en otras palabras, la gestión educativa tiene que ser la encargada de enlazar a todos los miembros, tanto internos como externos, para lograr que todos sean responsables, empoderados y capaces de participar de forma activa.

\section{CONCLUSIONES}

En conclusión, desde la gestión administrativa pareciera existir una separación entre la familia y la administración, pues no se encontraron acciones directas que se enfoquen en el apoyo del hogar para el estudiantado. Así, el alumnado se encuentra en medio de la familia, el personal docente, la persona orientadora y la dirección, todos involucrados, pero no enlazados para trabajar de forma integral. Por lo que se realiza una labor desvinculada, en otras palabras, si bien cada grupo trabaja por el mejoramiento del rendimiento académico, cada uno lo hace por su lado. No obstante, para lograr empoderar y vincular a todos los miembros de la comunidad educativa en el proceso de enseñanza-aprendizaje es necesario un gestor educativo interesado en conocer los problemas de la comunidad y en trabajar con soluciones que tomen en cuenta el contexto.

En cuanto a la familia, según la UNESCO (2004) existen diversos estudios realizados en América Latina que demuestran la importancia de acercar de forma activa a las personas encargadas al centro educativo, en este sentido señala que "el grupo de niños cuyos padres participaron como ayudantes de la maestra son los que lograron mejor rendimiento y sus madres, el mejor conocimiento de cómo apoyarlos" (p. 25). Aunque el texto solo lo ejemplifica en los primeros años de educación, es importante que sea un 
proceso continuo y fluido hasta el último año de secundaria con el fin de que sea un factor positivo para el mejoramiento del rendimiento académico.

Así bien, se afirma la necesidad de que la gestión administrativa busque estrategias para subir el rendimiento académico de una forma integral, o sea, que el centro educativo sea un asunto de toda la comunidad con una participación activa. Un medio inevitable para lograr el objetivo mencionado es la comunicación, pero mediante el diálogo, no a través de un monólogo.

Con el propósito de lograr pasar de la palabra a la acción, el director está obligado a educar y conscientizar a las madres y padres de familia en la importancia de su acompañamiento en la vida escolar. Además, el involucramiento será activo con el fin de apropiarse de la educación del alumnado.

También, mediante la investigación se concluye que la persona orientadora y la profesora guía tienen un trabajo de puente entre el hogar y el centro educativo, puente que debe evolucionar de una función informativa a una función impulsadora de la participación activa. Muchas veces el alumnado recurre a estas docentes como personas de confianza ante algún inconveniente dentro o fuera de la institución, por eso debe aprovecharse el rol de confidente para trabajar a profundidad de forma integral. La docente guía u orientadora para lograr constuir estos lazos necesita trabajar en conjunto con la dirección para planear, ejecutar y evaluar diferentes estrategias de empoderamiento de las familias desde las diversas falencias.

Finalmente, existe una intención e interés por parte de todos los actores por mejorar el rendimiento académico, pero las familias urgen del acompañamiento y la capacitación por parte del centro educativo para poder integrarse y mostrar una participación activa.

Es importante recalcar que la gestión educativa por realizar debe también acercar a la comunidad desde las primeras etapas de la educación, con el fin de obtener mejores resultados al finalizar el último año de colegio.

\section{REFERENCIAS}

Barquero, J. (2004). Cambios en las características y composición de las familias en Costa Rica y su vulnerabilidad frente a la pobreza. San José: Estado de la Nación. Recuperado de: http://estadonacion.or.cr/ files/biblioteca_virtual/010/Barquero_2004.pdf

Diario Oficial la Gaceta (6 de enero de 1988). Ley N 7739. San José: Imprenta Nacional.

Ministerio de Educación Pública. (2012). Informe Nacional de Factores Asociados al Rendimiento Académico en las Pruebas Nacionales Diagnósticas III Ciclo de la Educación General Básica 2010. San José, Costa Rica: Dirección de Gestión y Evaluación de la Calidad.

Oliva, E. \& Villa. V. (2014). Hacia un concepto interdisciplinario de la familia en la globalización. Justicia Juris, 10(1), 11-20. Recuperado de: http://search.ebscohost.com.talamanca.uned.ac.cr/login. aspx?direct=true \&db=sxi\&AN=101848751\&lang=es\&site=ehost-live

Programa Estado de la Nación. (2017). Sexto Informe Estado de la Educación. San José: Servicios Gráficos.

Ramírez, A. \& Rosés, P. (2005). Conciliación del trabajo y la vida familia. El caso de Costa Rica. San José: OIT. Recuperado de: http://www.ilo.org/wcmsp5/groups/public/---americas/---ro-lima/---sro-san_ jose/documents/publication/wcms_301562.pdf

Rico, A. (2016). La gestión educativa: Hacia la optimización de la formación docente en la educación superior en Colombia. Revista Sophia, 12(1) 55-70.

UNESCO. (2001). Declaración de Cochabamba. Recuperado de: http://unesdoc.unesco.org/images/0012/001214/121485s.pdf 
UNESCO. (2004). Participación de las familias en la educación infantil latinoamericana. Santiago, Chile: Editorial Trineo S.A.

UNESCO. (2011). Manual de gestión para directores de instituciones educativas. Recuperado de: http:// unesdoc.unesco.org/images/0021/002191/219162s.pdf 